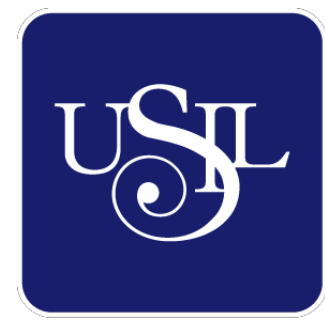

UNIVERSIDAD

SAN IGNACIO

DE LOYOLA

ESCUELA DE POSTGRADO

Maestría en Dirección de Marketing y Gestión Comercial

\title{
PLAN DE MARKETING PARA INCREMENTAR LA PARTICIPACIÓN DE MERCADO DE QLED TV EN EL SECTOR PREMIUM DE SAMSUNG ELECTRONICS PERÚ
}

Trabajo de Investigación para optar el grado de Maestro en Dirección de Marketing y Gestión Comercial

\section{DIEGO ALONSO BLANCO PINEDA}

ADRIÁN JESÚS RUITÓN CASTAÑEDA

\author{
Asesor: \\ Rodolfo Luis Gonzalez Angulo \\ Lima - Perú \\ 2018
}


A mi madre, quien a pesar de todas las adversidades nunca ha dejado de apoyarme y permitirme que este desafío hoy sea una realidad.

A Lindsay Valverde, por su ahínco en el trabajo y apoyo incondicional.

También quiero agradecer a quienes me ayudaron cuando más lo necesité, por extender su mano en momentos difíciles y por la amistad. De verdad mil gracias Celinda, Esther, María Inés y Hedy: siempre las llevaré en mi corazón.

Diego Alonso Blanco Pineda

Agradezco a mi padre y a mi madre que siempre me han apoyado a lo largo de mi vida y en la toma de decisiones correctas. Por todo el empeño que pusieron en mí, por el entusiasmo de salir adelante y ser mejor cada día. Gracias papá por ser un gran ejemplo a seguir y a mi madre por enseñarme que es ser perseverante y constante en esta vida. También agradezco a mis hermanos por su apoyo incondicional y por las palabras de aliento que supieron darme cuando más lo necesitaba.

En honor a la memoria de mis abuelos Roberto y Amparo que me miran desde el cielo y siempre los tengo presente. 


\section{PLAN DE MARKETING PARA INCREMENTAR LA PARTICIPACIÓN DE MERCADO DE QLED TV EN EL SECTOR PREMIUM DE SAMSUNG ELECTRONICS PERÚ}




\section{RESUMEN EJECUTIVO}

En el 2018, a meses que inicie el mundial de Rusia, se estima que en el mercado peruano las ventas en el sector de televisores, de la primera mitad del año, podrían llegar hasta el 65\% o incluso $70 \%$ por la atracción de la clasificación del equipo peruano al mundial. Normalmente, se vende hasta un 55\% de la venta en la primera mitad del año, pero la euforia de la clasificación peruana a un mundial después de 36 años podría incrementar aún más el porcentaje de la venta regular.

Adicionalmente, es importante considerar que la demanda de pantallas más grandes, con mayor resolución y lo equipos con mejor tecnología son cada vez más recurrentes, lo que impulsaría la categoría de televisores premium a un $8 \%$ de la venta total de televisores en el Perú.

Frente a esta coyuntura, es que nace el presente proyecto, el cual busca incrementar la participación de mercado de QLED TV en el sector Premium del 20\% a comienzos del 2018 al 30\% a comienzos del 2019. Logrando vender más de 5,000 unidades de televisores QLED e incrementar en un 50\% la venta de televisores mayores a 55 pulgadas.

Para lo cual se ha desarrollado el presente Plan de Marketing, que tiene como finalidad aplicar las estrategias necesarias para impulsar la venta de televisores QLED la categoría premium. Enfocando los esfuerzos en el punto de venta, ya que en el sector retail es determinante la decisión de compra en la categoría. 


\section{Índice General}

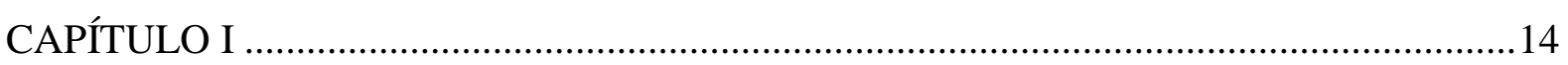

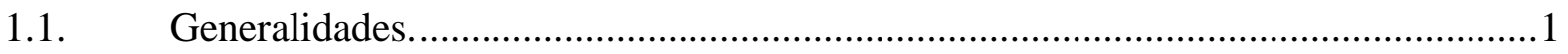

1.2. Antecedentes de la investigación. ................................................................. 1

1.3. Determinación del problema u oportunidad. .....................................................2

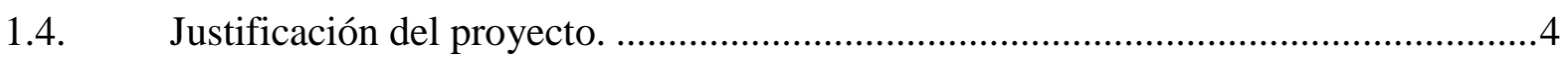

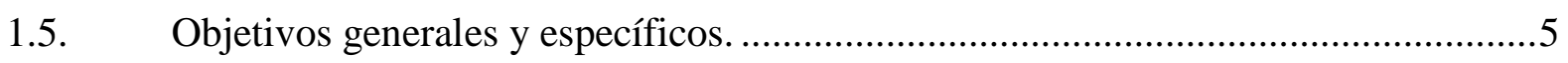

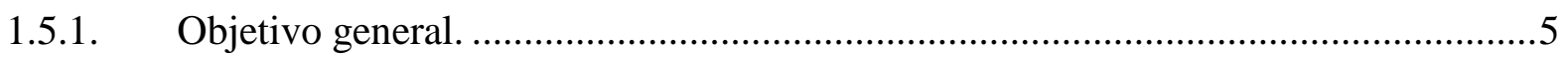

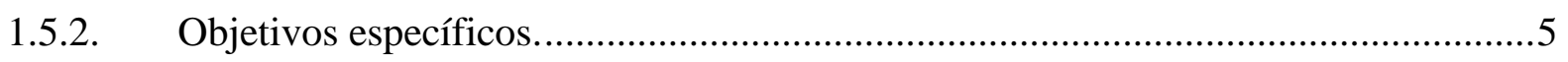

1.6. Alcances y limitaciones de la investigación. .............................................................

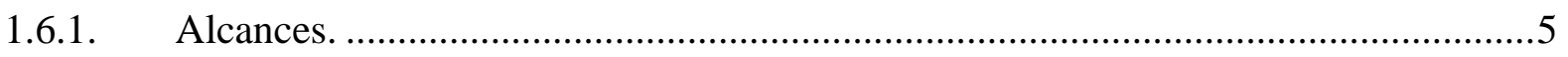

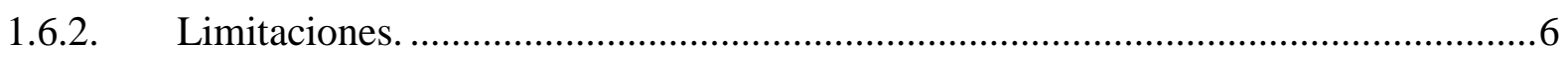

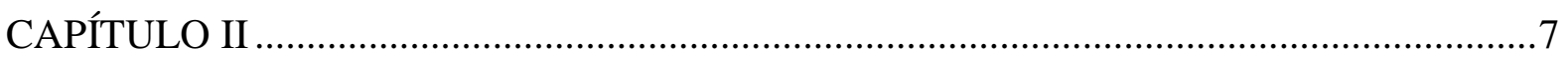

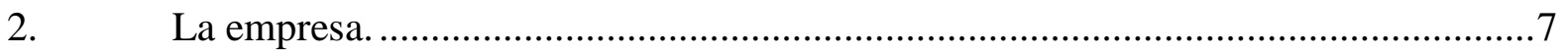

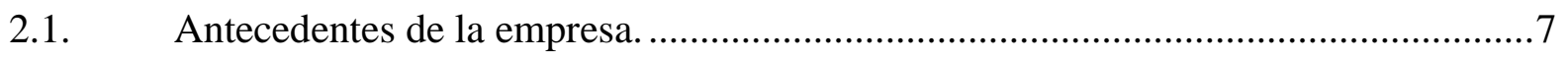

2.2. Estructura organizacional actual de la empresa. ............................................... 8

2.3. Situación de mercado y financiera actual de la empresa. ......................................

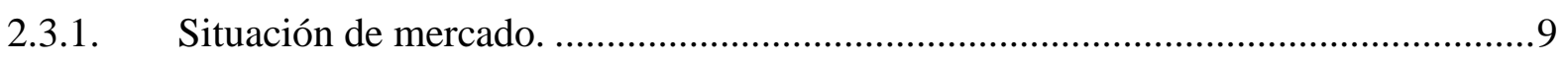

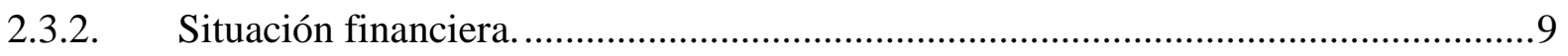

2.4. Misión, Visión y Valores de la Empresa. .............................................................11

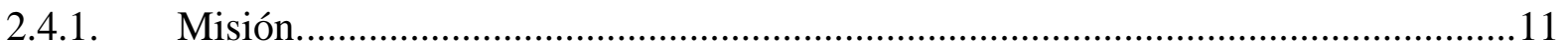

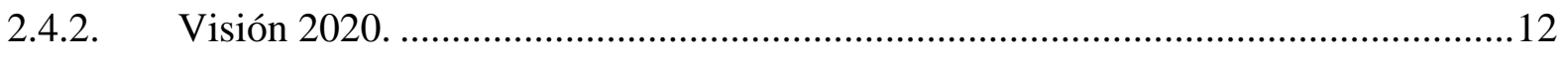

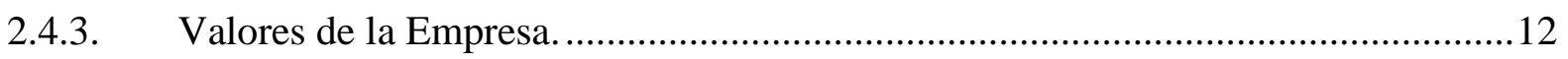




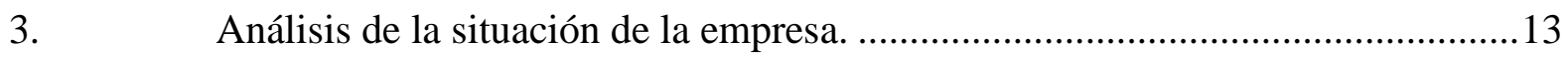

3.1. Análisis Externo: entorno, sector, mercado y competencia.................................... 13

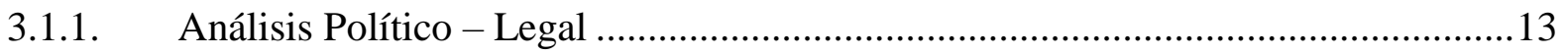

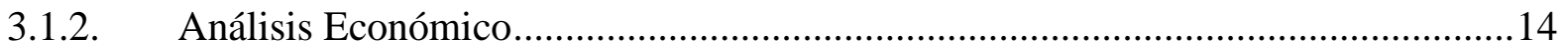

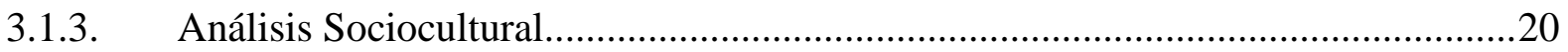

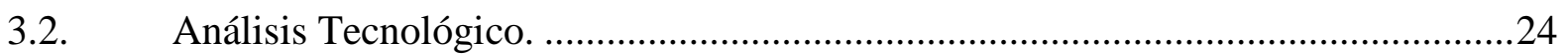

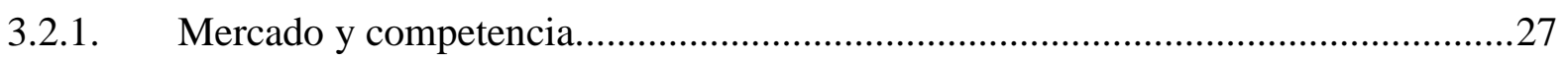

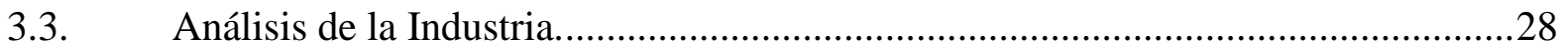

3.3.1. Demanda de televisores de pantalla grande UHD............................................28

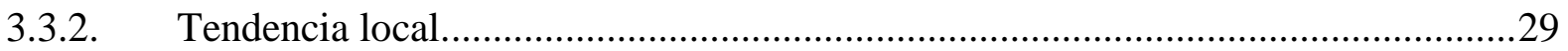

3.3.3. Descripción de las cinco fuerzas competitivas de la industria. ...............................31

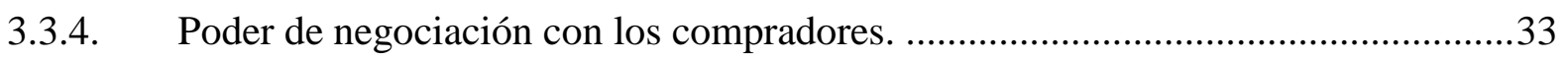

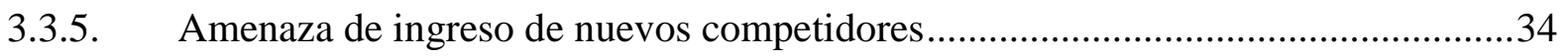

3.4. Poder de negociación con los proveedores ........................................................36

3.4.1. Análisis Interno: productos, ciclo de vida, precios, distribución, comunicación y fuerza de ventas

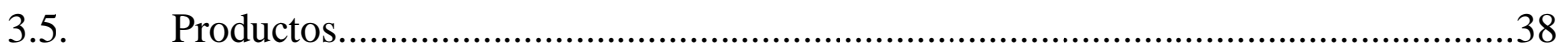

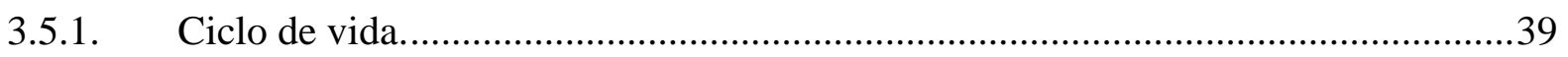

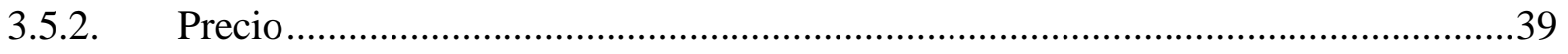

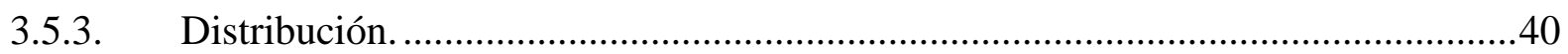

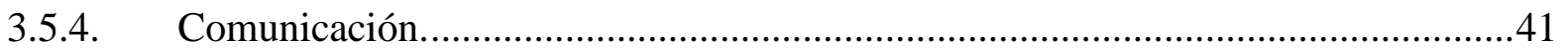

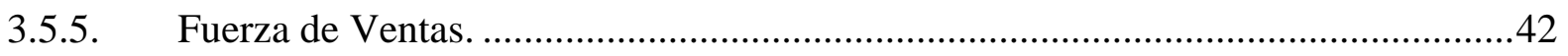

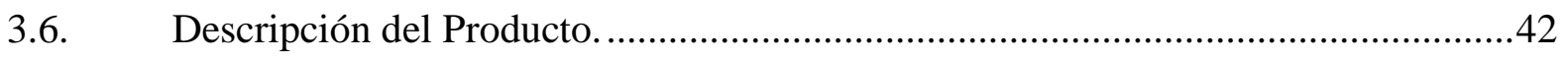

3.6.1. Competencias básicas de la empresa.................................................................... 


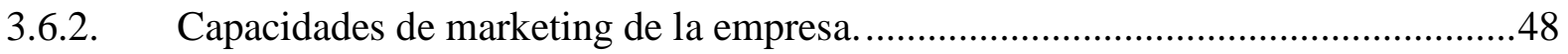

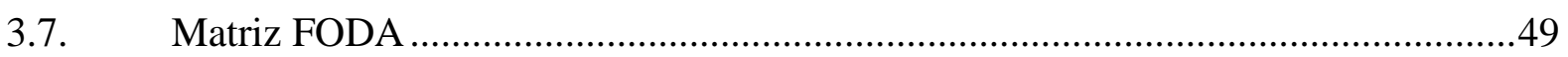

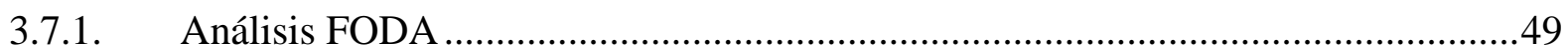

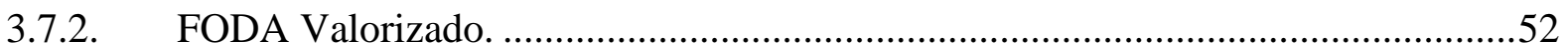

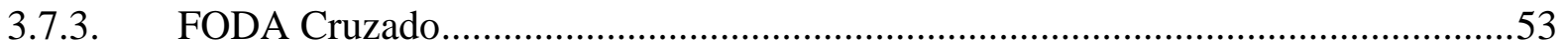

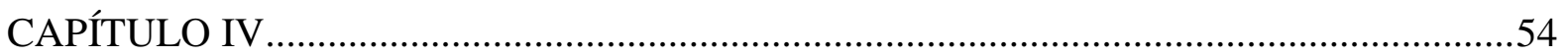

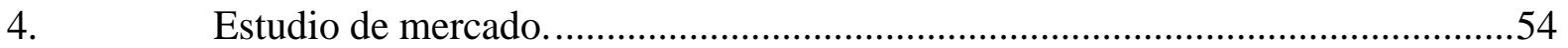

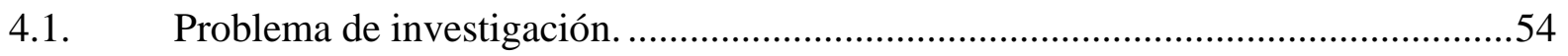

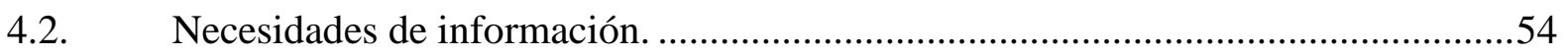

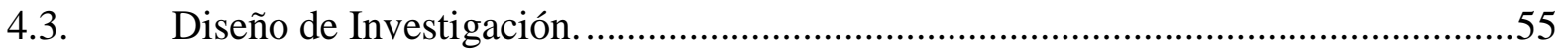

4.4. Método de investigación Cuantitativo ……..........................................................55

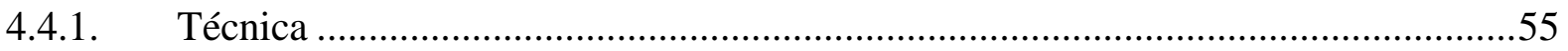

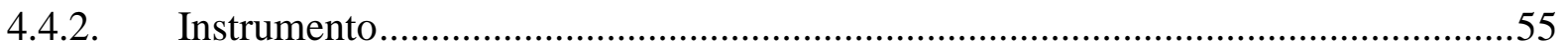

4.4.3. Proceso de selección de la muestra .......................................................................56

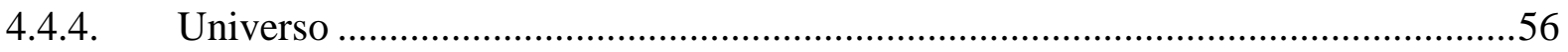

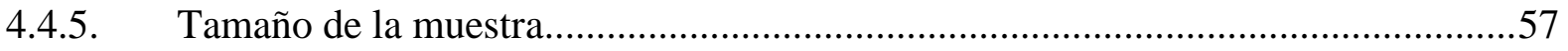

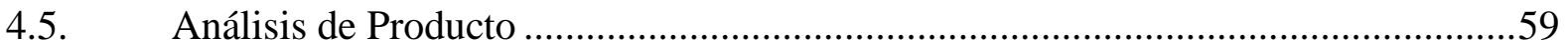

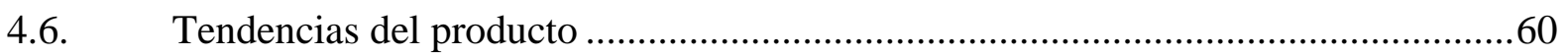

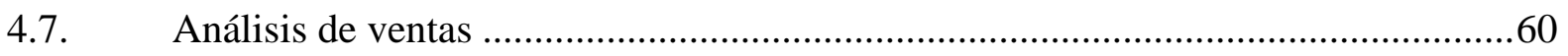

4.8. Tendencia del comportamiento del consumidor ...............................................63

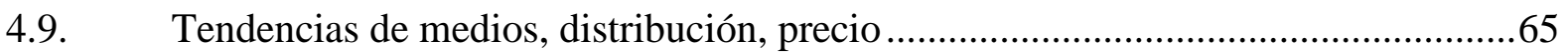

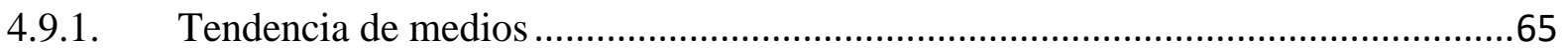

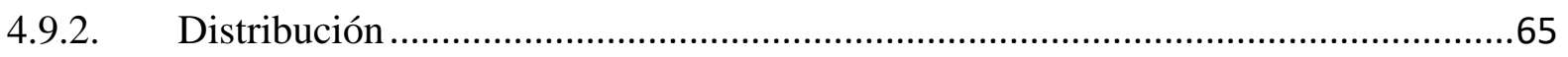

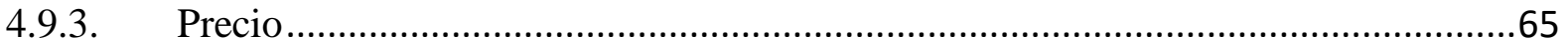

4.9.4. Segmentos del mercado que compra el producto (empresa e industria) .................66 
5. Objetivos.

5.1. Relación entre el Plan Estratégico y el Plan de Marketing...................................67

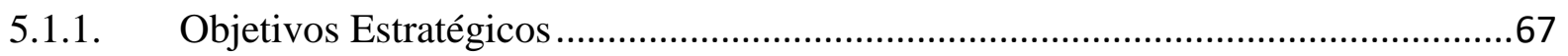

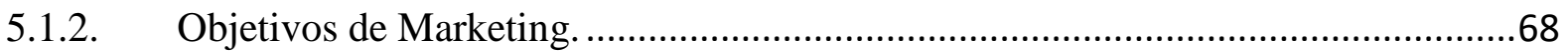

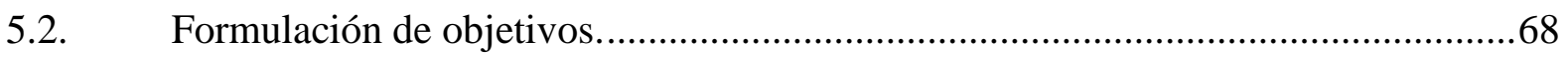

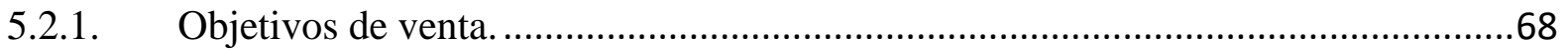

5.2.2. Objetivos de producto/ servicio.....................................................................68

5.2.3. Objetivos de comunicación. ...............................................................................69

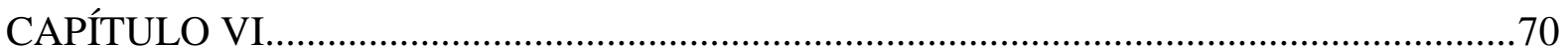

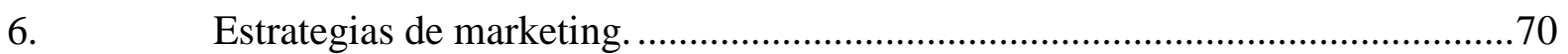

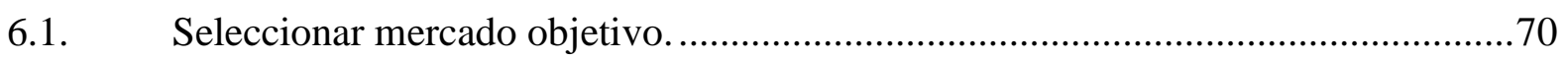

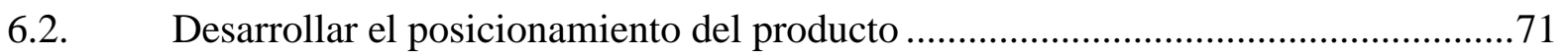

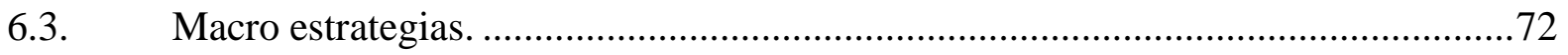

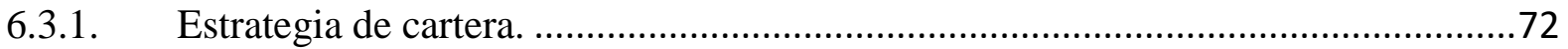

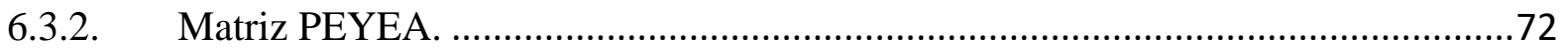

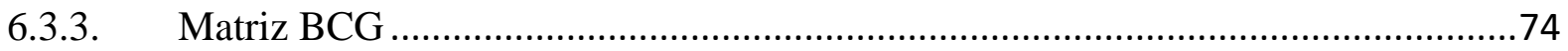

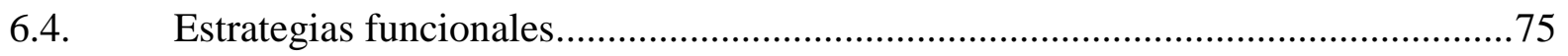

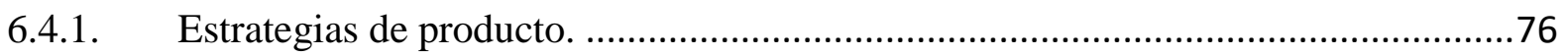

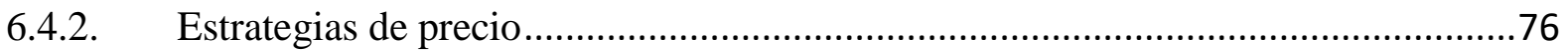

6.4.3. Estrategias de distribución y ventas ...............................................................76

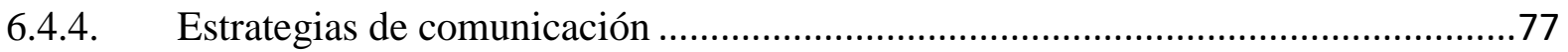

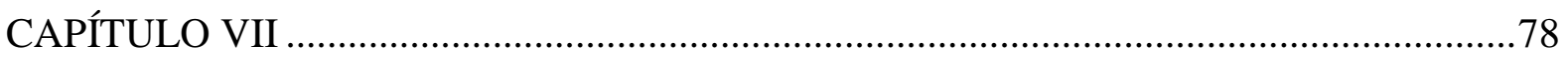

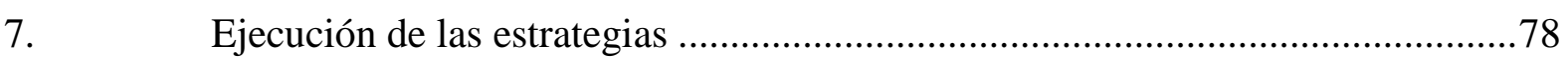




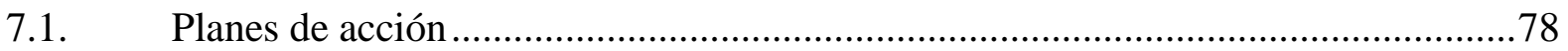

7.1.1. Plan de acción - Producto y Promoción..............................................................78

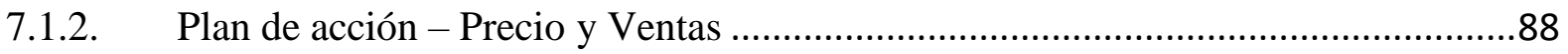

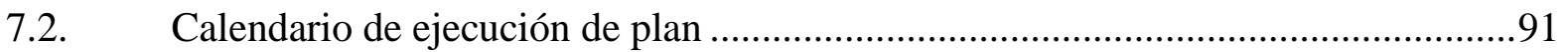

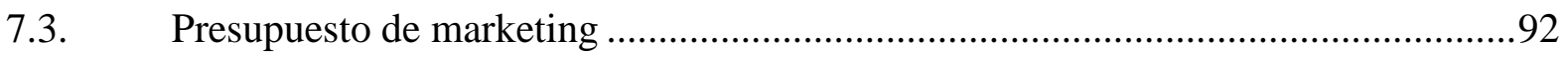

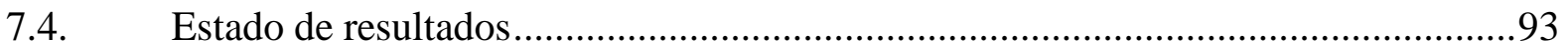

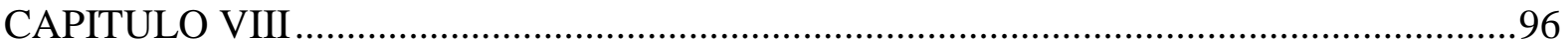

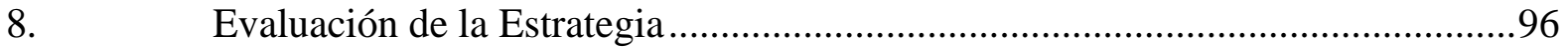

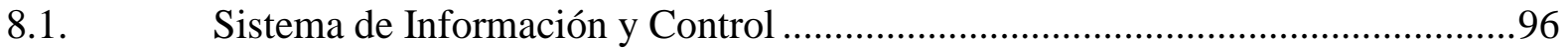

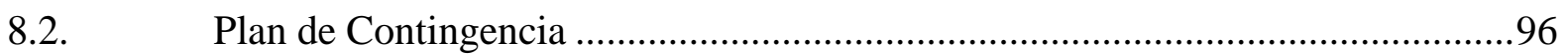

8.3. Evaluación financiera del plan................................................................. 98

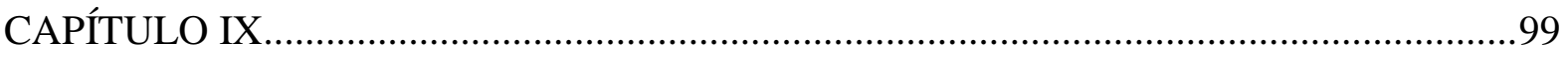

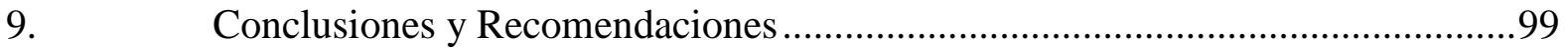

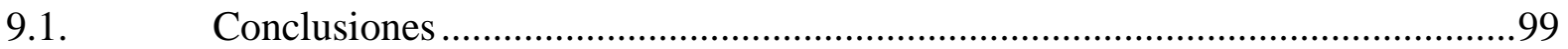

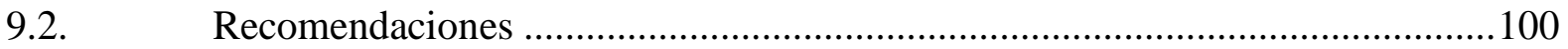

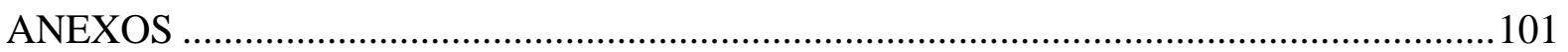

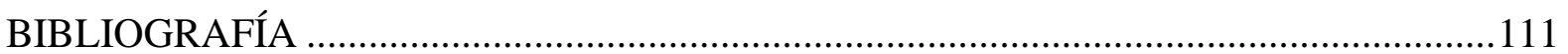




\section{Índice de Figuras}

Figura 1 Evolución del logotipo de Samsung. Elaboración propia 8

Figura 2 Organigrama SEPR 2017. Elaboración propia

Figura 3 Ingresos de Samsung Electronics Co., Ltd en miles de dólares americanos. 10

Figura 4 Ganancia de Samsung Electronics Co., Ltd en miles de dólares americanos. .11

Figura 5 Proyección, Reporte de Inflación, Panorama actual y proyecciones macroeconómicas

Figura 6 Proyección de la construcción de centros comerciales hasta el 2018. .17

Figura 7 Perú: Clase Media 2011-2015.

Figura 8 Peruanos que no han visto a Perú en una copa del mundo.

Figura 9 Principales desventajas al usar un televisor.

Figura 10 Participación de Mercado de Televisores - Perú.

Figura 11 Participación de Mercado de Televisores en sector Premium - Perú. .28

Figura 12. Matriz de competitividad de Porter.

Figura 13 Ciclo de vida del Producto QLED TV. .39

Figura 14 Comparación de colores del Televisor QLED frente a la competencia. .43

Figura 15 Comparativo de Tecnología HDR 1500 frente a otros televisores. .44

Figura 16 Conexión invisible del QLED frente a la competencia. .45

Figura 17 Diferencias entre la pantalla curva QLED y la pantalla Flat. .46

Figura 18. Un solo control remoto para manejar todos tus equipos. 
Figura 19. Preferencia de marca de televisor en casa.

Figura 20. Tamaño del Televisor de Preferencia en el Domicilio.

Figura 21. Factores que determinan la decisión de compra.

Figura 22. Modalidad de pago para adquirir un televisor.

Figura 23. Tiempo de renovación de televisores.

Figura 24. Preferencia de compra entre marca y tamaño de televisor. .64

Figura 25. Rango de precios de la compra de un nuevo televisor.

Figura 26. Mapa de Posicionamiento entre calidad y prestigio de marcas competidoras. .....71

Figura 27. Mapa de Posicionamiento entre calidad y prestigio de productos competidores...72

Figura 28. Samsung Electronics - Matriz PEYEA.

Figura 29. Samsung Electronics - Matriz BCG .75

Figura 30. Combo 1: QLED TV de 55” + Diseño de camiseta exclusiva de selección.

Figura 31. Combo 2: QLED TV de 65" + Diseño de camiseta exclusiva de selección + Celular Galaxy S8. .79

Figura 32. Combo 3: QLED TV de 75" + Diseño de camiseta exclusiva de selección + Celular Galaxy S8 + Soundbar Curvo. .79

Figura 33. Combo 1: QLED TV de 55" + Celular Galaxy A8. .80

Figura 34. Combo 2: QLED TV de 65" + Welcome Pack (Incluye Tablet Galaxy Tab A3)..80

Figura 35. Combo 3: QLED TV de 75" + Caja Welcome Pack (Incluye una Tablet Galaxy Tab A3) + Soundbar Curvo .80 
Figura 36. Combo 1: QLED TV de 55" + Netflix Gratis por 6 meses.

Figura 37. Combo 2: QLED TV de 65" + Netflix Gratis por 1 año.

Figura 38. Combo 3: QLED TV de 75" + Netflix Gratis por 1 año + Soundbar Curvo. .82

Figura 39. Propuesta de combo para la campaña "Renueva tu Hogar".

Figura 40. Propuesta de combo para la campaña "El regalo de tus sueños".

Figura 41. Ejemplo de activación en los Centros Comerciales. .84

Figura 42. Ejemplo de activación con redes sociales.

Figura 43. Ejemplo de post en redes sociales con activación. .86

Figura 44. Ejemplo de activación en el punto de venta.

Figura 45. Ejemplo de Merchandising en las activaciones. .87

Figura 46. Ejemplos de exhibición de material en el punto de venta. .88 


\section{Índice de Tablas}

Tabla 1

Tabla 2

Tabla 3

Tabla 4

Tabla 5

Tabla 6

Tabla 7

Tabla 8

Tabla 9 .90

Tabla 10

Tabla 11

Tabla 12

Tabla 13

Tabla 14

Tabla 15

Tabla 16

Tabla 17

Tabla 18 